\title{
GRANULOMATOSIS WITH POLYANGIITIS(WEGENER'S GRANULOMATOSIS); AN IMPORTANT RARE DIFFERENTIAL DIAGNOSIS FOR GENERALIZED HYPERPLASTIC GINGIVITIS.
}

Prasangi Madubhashini Peiris, D. LeukeBandara, P.V.K.S. Hettiarachchi, R.D.Jayasinghe, W.M.Tilakaratne.

1. Lecturer in Oral Medicine and Radiology, Department of Oral Medicine and Periodontology, Faculty of Dental Sciences, University of Peradeniya, Sri Lanka.

Contact number+94718176105, E-mail : prasangipeiris@gmail.com

2. Senior lecturer in Periodontology, Department of Oral Medicine and Periodontology, Faculty of Dental Sciences, University of Peradeniya, Sri Lanka.

Contact number +94-81-2 397458, E-mail: $\underline{\text { dhanulb@yahoo.com }}$

3. Senior lecturer in Oral Medicine and Periodontology, Department of Oral Medicine and Periodontology, Faculty of Dental Sciences, University of Peradeniya, Sri Lanka.

Contact number+94-81-2 397458, E-mail: kalaniz2004@yahoo.com

4. Chair Professor of Oral Medicine and Periodontology, Department of Oral Medicine and Periodontology, Faculty of Dental Sciences, University of Peradeniya, Sri Lanka.

Contact number+94-81-2 397451, E-mail: ruwanduminda@yahoo.com

5. Professor of Oral Pathology, Department of Oral and Maxillofacial Clinical Sciences, Faculty of Dentistry, University of Malaya, Malaysia.

Contact number+94773438477, E-mail:wmtilak@um.edu.my

\section{Corresponding Author:}

W.M.Tilakaratne,

Department of Oral and Maxillofacial Clinical Sciences,

Faculty of Dentistry, University of Malaya, Malaysia.

Contact number-+94773438477

E-mail ID :wmtilak@um.edu.my

Submitted Date :31-12-2019

Accepted Date :22-01-2020

\begin{abstract}
Granulomatosis with polyangiitis (GPA) formerly known as Wegener's granulomatosis is a rare lifethreatening disease which can affect multiple organs of the body including oral cavity. The oral manifestation of GPA has been named as strawberry gingivitis due to its characteristic features. We report a case of GPA in a 68-year-old lady who presented with Strawberry gingivitis as the initial symptom and successfully managed with prednisone and cyclophosphamide. This case demonstrates an orofacial manifestation of a systemic disease which emphasises the importance of awareness of this kind of manifestations among dental professionals in order to facilitate early diagnosis.
\end{abstract}

Key words: Anti neutrophil cytoplasmic antibody, Granulomatosis with polyangiitis, Strawberry gingivitis. 


\section{INTRODUCTION}

Granulomatosis with polyangiitis (GPA) is a rare but potentially life threatening disease which manifests as characteristic gingival lesions where dentists should play an important role in early diagnosis and management. GPA was previously known as Wegener's Granulomatosis and new terminology was introduced according to the recommendation of the Boards of Directors of the American College of Rheumatology, the American Society of Nephrology and the European League Against Rheumatism for a shift from honorific eponyms to disease descriptive or etiology- based nomenclature.' GPA is considered as a systemic disease due to small vessel vasculitis but the exact aetiology is yet to be understood. Klinger described this disease as a variant of polyarteritisnodosa in 1931 and Friedrich Wegener described the disease as a separate syndrome for the first time in 1936 using autopsy findings of a 38 year old man. ${ }^{2}$ For the second time Wegener reported the disease in 1939. The term 'Wegener's Granulomatosis " was introduced by Godman and Churg in 1954 and at the same time established the below mentioned criteria to diagnose the disease :1) granulomas in the upper respiratory tract; 2)necrotising vasculitis; and 3)glomerulonephritis. ${ }^{3}$ It has been estimated that the incidence of GPA is 8-10 cases per one million depending on geographic location. ${ }^{4}$ It usually occurs in old age and it has been reported to have a peak incidence among patients between 64 75 year old. ${ }^{4.5}$ There is no gender predilection among reported cases of GPA. GPA has a wide spectrum of clinical presentation where respiratory system and kidneys are most commonly affected. Because of this variation, the concept of limited GPA (wegener's) was introduced to describe the locoregionally affected disease.

\section{Case Report}

A 68 year old lady presented to the Oral Medicine clinic ,University Dental Hospital, complaining a gingival over growth and bleeding from gingiva. The history of the gingival overgrowth as revealed by the patient had been to commence from a papilla of a lower tooth which has gradually expanded in to buccal,palatal and lingual gingivae. However, there was no involvement of other oral soft tissues. Although the patient has sought medical and dental advice for her condition several times, it had progressed despite the treatments she underwent. Finally a general practitioner referred her to the Oral Medicine clinic at the University hospital. At the initial presentation to the Oral Medicine clinic, the patient was suffering from reddish gingival over growth with bleeding from gingival tissues(Figure 1) and difficult to eat due to bleeding and pain. On examination she had enlarged cervical lymph nodes which were tender(Figure 2). Basic periodontal examination revealed code 3 as the highest code despite the severe bleeding observed during probing. She was on regular medication for hypertension and dyslipidaemia. Patient was admitted to the University Dental Hospital ward for investigations and management of her condition. On admission she was febrile but there were no other significant signs or symptoms indicative of other systemic illnesses.

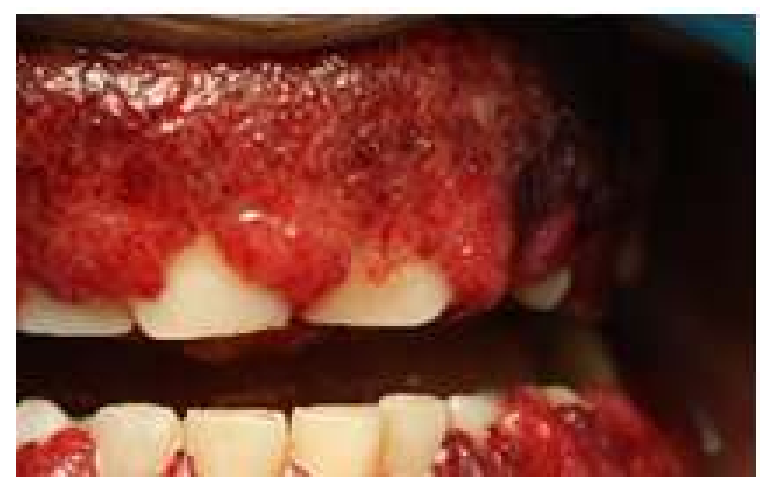

Fig 1. Initial presentation of the patient with strawberry gingivitis affecting both upper and lower gingivae.



Fig 2. Bilateral enlarged tender cervical lymph nodes due to secondary infection of the gingival lesions

Granulomatosis with polyangiitis and haematological malignancy were considered as differential diagnoses for her condition. All the routine investigations were performed with $\mathrm{C}$ reactive protein (CPR), Erythrocyte sedimentation rate (ESR), blood picture, chest radiograph, renal function test, liver function test(LFT), urine full report(UFR), Ultra sound scan(USS) of bilateral neck, USS of abdomen and 2D ECHO. An 
incisional biopsy and Antineutrophil cytoplasmic antibody(ANCA) test were performed as special investigations in order to confirm the clinical diagnosis. Patients white blood cells (WBC) count was high $\left(17.83 \times 10^{3} / \mathrm{uL}\right)$ with differential counts as following - Neutrophils 13.68 x $10 \%$ uL , lymphocytes $-3.25 \times 10 \% \mathrm{uL}$ and blood picture report excluded the possibility of acute leukaemia, however, reported elevated WBC count compatible with bacterial infection with coexisting iron deficiency anaemia.ESR was significantly high and Chest radiograph, USS abdomen, LFT, UFR and 2D ECHO were normal. Meanwhile histopathological diagnosis came as Wegener's granulomatosis and ANCA test was suggested to confirm the diagnosis. The microscopic findings were mucosa covered by hyperplastic parakeratinized stratified squamous epithelium and corium containing a dense neutrophil predominant inflammatory infiltrate with eosinophils and scattered giant cells with neutrophil abscesses and small vessel vasculitis. The features were compatible with Wegener's granulomatosis (Figures 3,4,5).
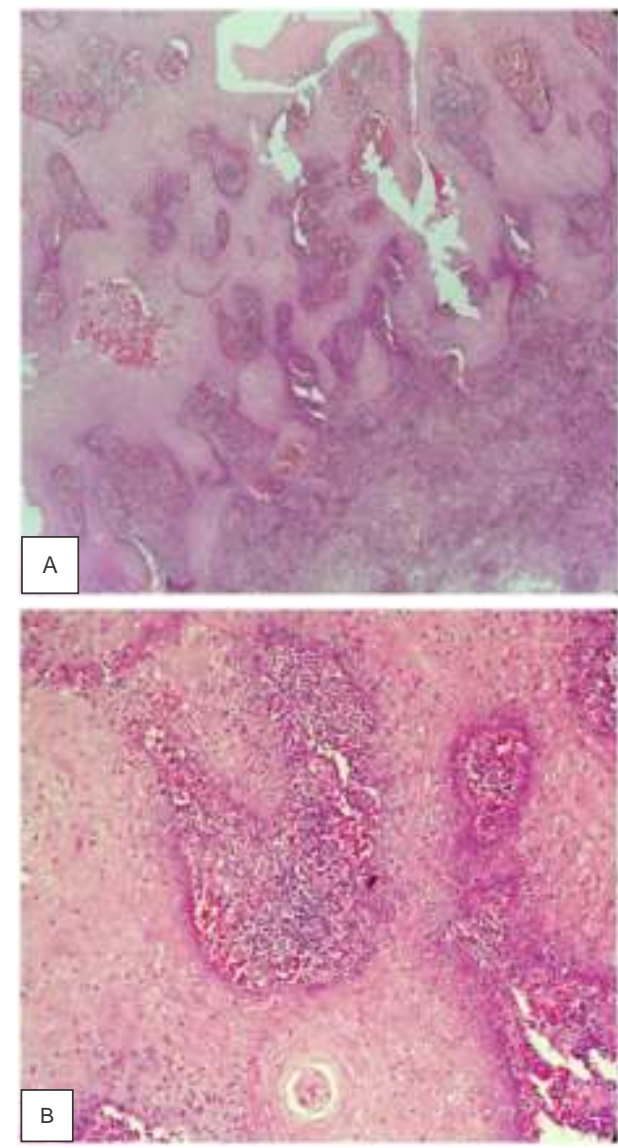

Fig 3. Gingival biopsy showing pseudoepitheloimatous hyperplasia(A) and presence of neutrophil abscess(B) in Hematoxylin and Eosin section

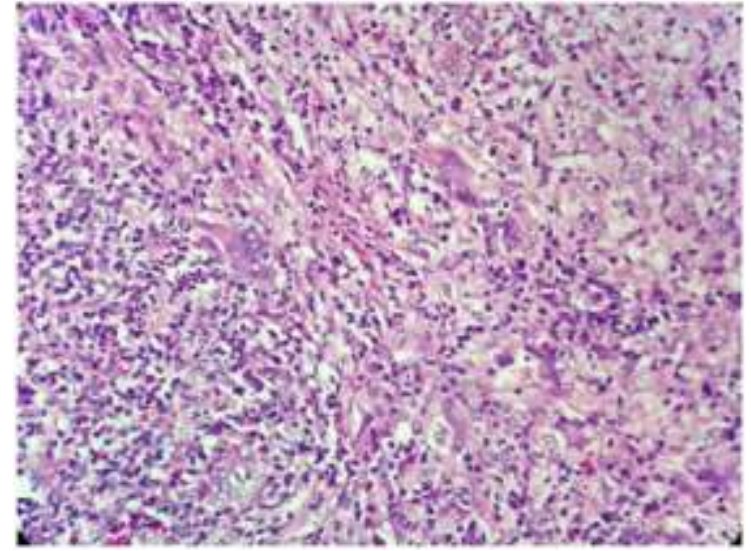

Fig 4. Presence of mixed population of inflammatory cells with occasional giant cells

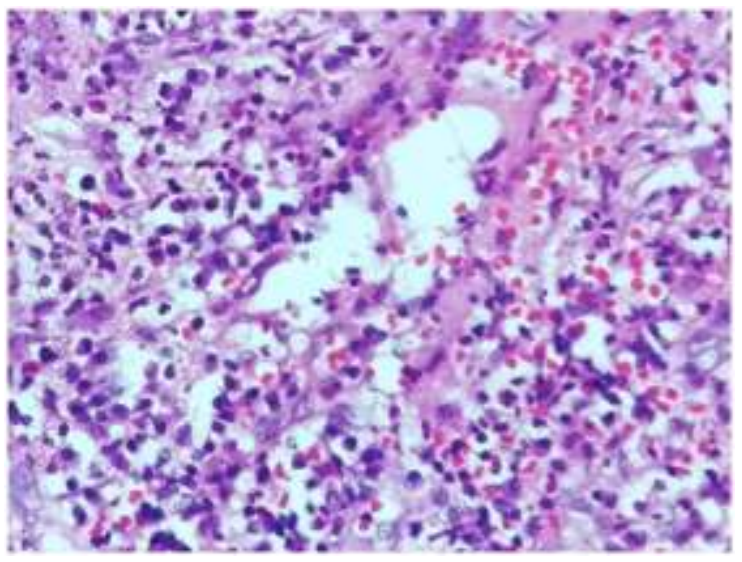

Fig 5. Small vessel vasculitis with inflammatory cell infiltration

The ANCA test result was positive confirming the histopathological diagnosis of Wegener's granulomatosis or Granulomatosis with polyangiitis. Patient was seen by the physician and involvement of other organs was excluded with the help of clinical features and findings of the investigations. Treatment for GPA was started with Prednisone $60 \mathrm{mg}$ and cyclophosphamide 100mg daily doses. At the same time she was treated for iron deficiency anaemia with ferrous sulphate, vitamin B,C and folic acid and with broad spectrum antibiotics for secondary infection. Her random blood sugar level and renal functions were regularly monitored. Patient responded well to the treatment and there was marked clinical improvement with reduction in bleeding and over growth of the gingiva with time(Figure 6,7). 


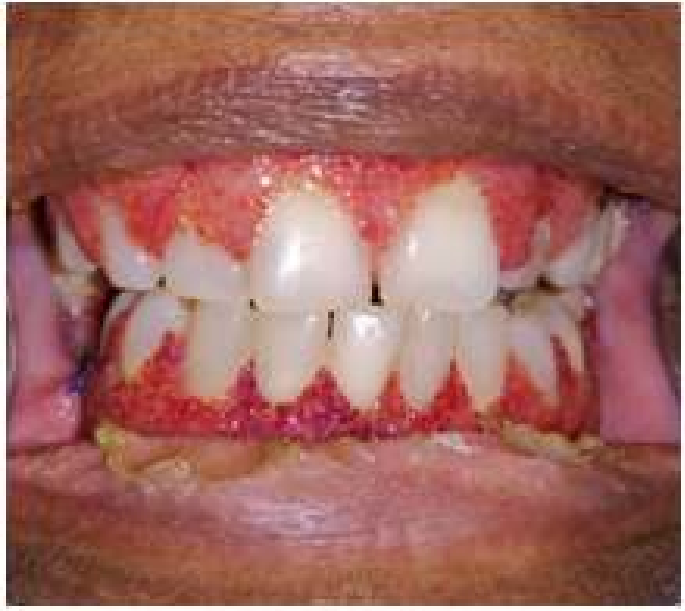

Fig 6. Four days after the systemic drug therapy (prednisone and cyclophosphamide) and periodontal debridement

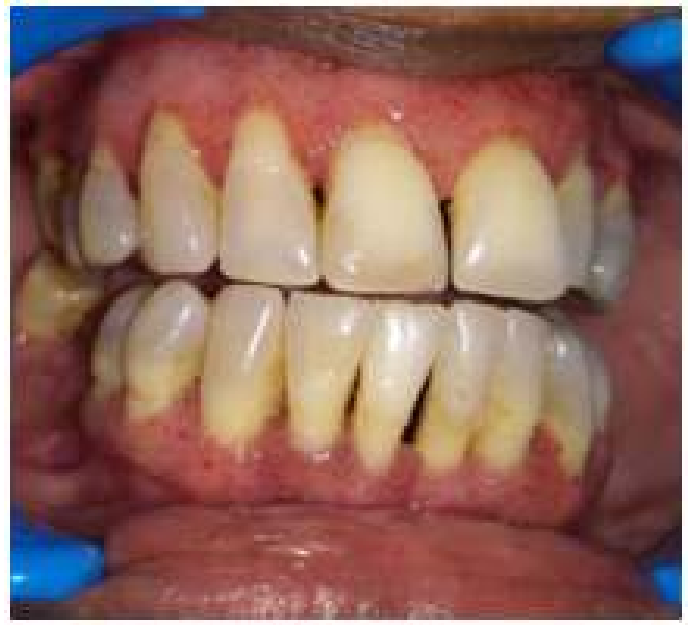

Figure 7. Resolution of features of Strawberry gingivitis three weeks after the therapy

The objective improvement of the condition was monitored with the ESR levels, which also improved with time. Plaque control measures were introduced and the patient underwent periodontal debridement following settlement of the acute presentation. Patient was recovering from pain and she started to have solid meals gradually. After two weeks of prednisolone and cyclophosphamide therapy as an inward patient she was discharged with reduced doses of prednisone (40mg daily dose) and cyclophosphamide(50mg daily dose). The patient was reviewed in one week at the Oral Medicine clinic and was decided to replace cyclophosphamide with Azathioprine. Currently the patient is under regular follow up.

\section{DISCUSSION}

GPA is a disease categorized as systemic vaculitides associated with anti-neutrophil cytoplasmic autoantibodies? It is a rare disease which can affect multiple systems of the body particularly the respiratory tract including sinuses , nose, trachea, lungs, kidneys, nervoussystem, eyes, ears, heart, skin and oral cavity. Due to multi organ involvement, clinical presentation of GPA may have a wide variation. Persistent-recurrent nasal discharges, epistaxis, nasal bridge collapse, mucosal ulceration and sinus inflammation are some of the clinical features indicating involvement of nose and paranasal sinuses. Cough, breathlessness, stridor, wheeze, small air way obstruction, pulmonary nodules, cavitating lung lesions, pleuritis, pleural effusions, pulmonary haemorrhage and respiratory failure are the suggestive features of lower airway involvement. Clinical features indicative of kidney involvement are haematuria, proteinuria, cellular casts in urine cytology, renal impairment manifesting as acute kidney injury, chronic kidney disease or end stage renal failure. ${ }^{3} \quad$ Oral ulcers,oral granulomatous lesions and gingival hyperplasia called "Strawberry gingivitis" are some of the oral manifestations of GPA. Strawberry gingivitis is considered as a pathognomonic feature of GPA. Erythematous hyperplastic gingiva with reddish or yellowish spots looks like the outer surface of ripe strawberry has lead to use this terminology. The concept of limited GPA was introduced in 1953 by Fienberg to describe the locoregionalpresentationof GPA. The reported frequency of presence of oral lesions vary from 10 to 62 percent $^{8}$ but only 5 to 6 percent of patients have oral lesions as initial sign., ${ }^{2,9}$ Other than the characteristic strawberry gingivitis, tooth mobility, impaired healing after extraction, cranial nerve palsies and parotid gland swelling can occur in orofacial region.

Even though the aetiology of GPA has not been clearly understood it is considered to be of autoimmune in origin and triggered by an environmental insult in a genetically susceptible patient. ${ }^{5}$ It is believed that both cell-mediated and humoral axis of immune system involve in granuloma formation and vasculitis of GPA. Production of Tumour necrosis factor alpha(TNF$\alpha)$ and Interferon gamma by T-cells contributes to the inflammatory process and neutrophils also play a significant role in tissue injury of GPA patients. Expression of antigens on activated neutrophils is 
stimulated by inflammatory cytokines such as TNF$\alpha$ which will lead to interact with antineutrophil cytoplasmic antibody(ANCA). It can cause degranulation of neutrophils and production of toxic products. Those toxic products cause tissue injury. ${ }^{2,5}$ Testing for positivity of ANCA will aid the diagnosis of GPA. There are two patterns of ANCA; cytoplasmic ANCA(c-ANCA) and perinuclear ANCA(p-ANCA). c- ANCA is associated with autoantigen protease 3 (PR3) which is a protease in granules of neutrophils. It is considered that cANCA is highly specific for GPA and also its titres are indicative of disease activity.

In 1990 American College of Rheumatology introduced diagnostic criteria for GPA. ${ }^{2}$ Presence of at least two of the four criteria will facilitate the diagnosis of GPA with an $88 \%$ sensitivity and $92 \%$ specificity. ${ }^{2,3}$ The criteria are as follow i)oral ulcers or nasal discharges ii) abnormal findings on the chest radiograph iii)A urinary sediment containing red blood cell casts or more than five red blood cells per high-power field and iv) granulomatous inflammation on biopsy.

Inclusion of oral manifestations of GPA in to the above criteria indicates their importance in diagnosing the disease. Therefore, dental professionals play a significant role in the diagnosis and management since patients initial presentation may be oral lesions or gingivitis. Consideration of GPA as a possible diagnosis in patients with characteristic strawberry gingivitis or even with non-specific oral lesions will help in early diagnosis leading to reduce morbidity and mortality caused by GPA. Management of GPA should be a multidisciplinary approach. Medical management is provided with combination of glucocorticoids and cyclophosphamide initially and azathioprine or methotrexate can be used in the maintenance phase instead of cyclophosphamide. ${ }^{3,9}$ Other than the systemic drug therapy, local therapies should be provided according to the systems involved. Thus, periodontal debridement with adjunctive use of antibacterial mouth rinses and emphasize on the plaque control measures could be included under local measures in the overall management of patients presented with oral lesions.

\section{CONCLUSION}

Awareness on Orofacial manifestations of systemic diseases is important for dental professionals as they could be the first in identifying those systemic diseases. This could facilitate early diagnosis and early intervention reducing morbidities and mortality that could result from the systemic disease.

\section{ACKNOWLEDGMENT}

The authors are thankful to the patient who gave consent for the publication of this case report.

\section{REFERENCES}

1. Falk RJ, Gross WL, Guillevin L, Hoffman GS, Jayne DRW, Jennette JC, et al. Granulomatosis with polyangiitis (Wegener's): An alternative name for Wegener's granulomatosis. Arthritis Care Res. 2011;63(4):863-4.

2. Stewart C, Cohen D, Bhattacharyya I, Scheitler L, Riley S, Calamia K, et al. Oral manifestations of Wegener's granulomatosis: A report of three cases and a literature review. J Am Dent Assoc [Internet]. 2007;138(3):338-48. Available from: http://dx.doi.org/10.14219/jada.archive.2007.01 66

3. Greco A, Marinelli C, Fusconi M, Macri GF, Gallo A, De Virgilio A, et al. Clinic manifestations in granulomatosis with polyangiitis. Int $\mathrm{J}$ Immunopathol Pharmacol. 2016;29(2):151-9.

4. Ntatsaki E, Watts RA, Scott DGI. Epidemiology of ANCA-associated vasculitis. Rheum Dis Clin North Am. 2010 Aug;36(3):447-61.

5. Kubaisi B, Samra KA, Foster CS. Granulomatosis with polyangiitis (Wegener's disease): An updated review of ocular disease manifestations. Intractable Rare Dis Res. 2016;5(2):61-9.

6. Szczeklik K, Wludarczyk A, WawrzyckaAdamczyk K, Górka J, Fuks-Kulska M, Darczuk $\mathrm{D}$, et al. Oral manifestations of granulomatosis with polyangiitis - Clinical and radiological assessment. J Dent Sci. 2019;14(1):54-60.

7. Moiseev S V. Classification, diagnosis and treatment of ANCA-associated vasculitis. World J Rheumatol. 2015;5(1):36.

8. Patten SF, Tomecki KJ. Wegener's granulomatosis: Cutaneous and oral mucosal disease. J Am Acad Dermatol. 1993;28(5):710-8.

9. Sung IY, Kim YM, Cho YC, Son JH. Role of gingival manifestation in diagnosis of granulomatosis with polyangiitis (Wegener's granulomatosis). J Periodontal Implant Sci. 2015;45(6):247-51. 